\title{
Associated Standard Model Higgs boson search with ATLAS
}

\section{Inga LUDWIG* on behalf of the ATLAS collaboration}

University of Freiburg, Germany

E-mail: inga.ludwig@cern.ch

\begin{abstract}
The associated Standard Model Higgs boson production channels have the lowest cross-sections among the leading production processes at the LHC. Nevertheless, in the low Higgs boson mass range, the associated production of a Higgs boson with a $t \bar{t}$-pair might contribute to the Higgs boson discovery. Furthermore, it can contribute valuable information in the measurement of the Higgs boson properties, in particular about the $t \bar{t} H$ Yukawa coupling. A Monte Carlo study of the channel $t \bar{t} H, H \rightarrow b \bar{b}$ in the semi-leptonic final state as well as the results of feasibility studies for the $H \rightarrow W W$ decay mode of $t \bar{t} H$ and $W H$ at the ATLAS experiment are presented here.
\end{abstract}

Physics at LHC 2008

29 September - October 4, 2008

Split, Croatia

\footnotetext{
* Speaker.
} 


\section{Introduction}

The associated Standard Model Higgs boson production channels have the lowest cross-sections among the most important production processes at the LHC. In the low Higgs mass region between 115 and $130 \mathrm{GeV}$, where the Higgs boson preferentially decays into a pair of $b$-quarks, the associated production with a top quark pair may contribute to a discovery. The observation of the Higgs boson in the $H \rightarrow b \bar{b}$ decay mode via the gluon fusion and vector boson fusion (VBF) processes is hopeless, due to the large background from jet production from QCD processes. In contrast, the signature of the accompanying top quarks in $t \bar{t} H$ production allows to trigger the channel and to better suppress the large backgrounds. In the intermediate mass region around $160 \mathrm{GeV}$, the decay in two $W$ bosons is dominant and a discovery is expected through gluon fusion or VBF. Once the Higgs boson has been observed, the associated production channels will become important, since they provide access to the Higgs boson's gauge- and Yukawa-couplings.

This article covers studies of the $b \bar{b}$ and $W W$ final states of $t \bar{t} H$ production and the $W W$ final state of production in association with a $W$ boson. Studies of these and other decay modes of associated production at ATLAS are described in detail in [1]. All studies have been performed with Monte Carlo samples, using a detailed simulation of the ATLAS detector and assume an integrated luminosity of $30 \mathrm{fb}^{-1}$.

\section{2. $t \bar{t} H, H \rightarrow b \bar{b}$}

The most promising final state of the $H \rightarrow b \bar{b}$ channel is the decay mode $t \bar{t} H \rightarrow \ell v q q^{\prime} b \bar{b} b \bar{b}$, where the charged lepton can be used to trigger the channel. This final state contains four $b$ jets from the top and Higgs boson decays. Since it can be reconstructed completely, it offers the opportunity to suppress backgrounds, which consist mainly of non-resonant $t \bar{t} b \bar{b}$ (irreducible) and $t \bar{t}+$ jets production (reducible background). Other backgrounds, such as $W+\mathrm{jets}, W t$ or jet production via QCD processes have not been studied yet, but are expected to be negligible due to the reconstruction of the $t \bar{t}$ system. This needs to be confirmed in future studies.

A large fraction of the $t \bar{t}$ background is suppressed by a set of preselection cuts, including trigger requirements and off-line criteria that ensure the presence of exactly one isolated and identified electron or muon and at least six jets with $p_{T}>20 \mathrm{GeV}$, which are reconstructed by a $\Delta R=0.4$ cone algorithm. At least four jets need to be identified by a $b$-tagging algorithm based on the lifetime of the $b$-hadron [2], with only the four jets with the largest $b$-tag weights assigned by the algorithm being treated as $b$-jets. The suppression of $t \bar{t}$ achieved by the preselection is roughly two orders of magnitude larger than that for the signal and irreducible backgrounds.

To achieve further reduction of all backgrounds, a reconstruction of the $t \bar{t}$ system is attempted. The simplest approach is based on a set of cuts, starting from a reconstruction of the hadronic $W$ boson from pairs of light jets. The neutrino from the leptonic $W$ decay is reconstructed from the measured missing transverse energy $\left(\mathbb{E}_{T}\right)$ under the assumption of the $W$ boson being produced on-shell. The $W$ candidates are then paired with $b$-jets to obtain a set of top quark candidates, with mass window cuts on the hadronic $W$ boson and the top masses, serving to reduce the number of combinations to be considered. For these combinations a $\chi^{2}$ is defined to evaluate the deviation of the reconstructed top masses from their nominal values. The combination with the smallest $\chi^{2}$ - 
value is selected and the two remaining $b$-jets are assigned to the Higgs boson decay. Figure 1 (left) shows the resulting invariant $b \bar{b}$ mass distribution for the signal. The fraction of about $30 \%$ of all events for which the correct jets were assigned to the Higgs boson decay is indicated separately. The Higgs boson mass resolution that can be achieved by this approach is $22.8 \mathrm{GeV}$. In the plot on the right, which shows the signal stacked on top of the background, no clean mass peak is visible on the large backgrounds. Nevertheless, the signal-to-background ratio, $S / B$, can be improved by a mass window cut around the nominal Higgs boson mass. The working point for this analysis was chosen such that a signal cross-section of about $1 \mathrm{fb}$ is accepted and $S / B$ rises from about $5 \%$ to $11 \%$, compared to the preselection.
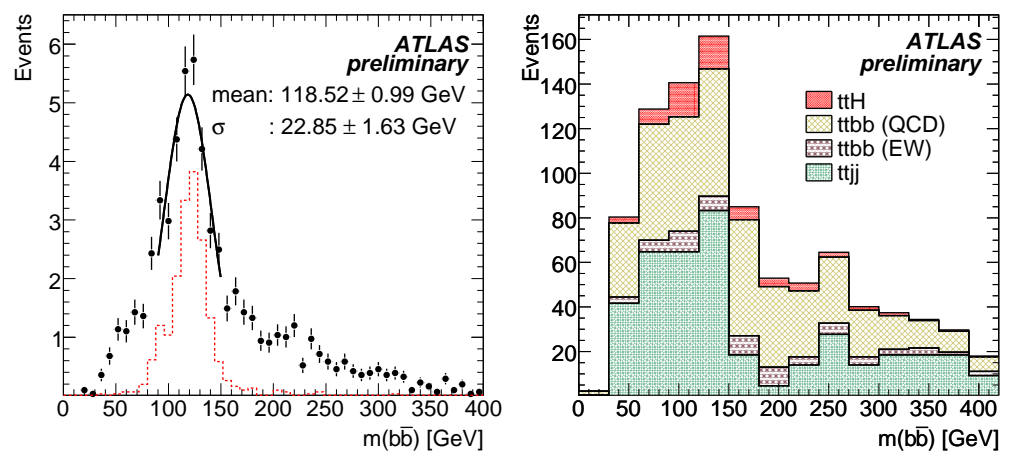

Figure 1: Invariant $b \bar{b}$-mass distributions. (Left): Signal only, the dashed red histogram contains only events where the correct jets were assigned to the Higgs boson decay. (Right): All samples, showing the contributions stacked.

Further improvement is expected from the use of multivariate methods to reject combinatorial and other backgrounds. The so-called pairing likelihood approach exploits kinematic variables of the top quark system as input to a combinatorial likelihood. Another method makes use of a constrained fit to adjust the jet momenta and $\mathscr{E}_{T}$, such that the $W$ and top masses are matched. The resulting four-momenta are used to calculate a $\chi^{2}$, which is a strong discriminating variable that is used as one of several inputs for a two-step likelihood, trained to reduce both, combinatorial and other background. The signal and background conditions achieved at the working points of the three analyses are summarised in Table 1 . The significance, $S / \sqrt{B}$, is quoted without taking systematic uncertainties into account. In addition, the fraction of events is given for which the jets associated to the Higgs boson and all four $b$-jets are assigned correctly. The maximum fraction of events for which the jets associated to the Higgs boson $(b \bar{b}$ purity) and all four $b$-jets are assigned correctly is reached in the pairing likelihood analysis at $34.0 \%$ and $27.5 \%$, respectively [1].

Fig. 2 (left) compares the ability of the three approaches to choose the correct combination of $b$-quark jets in terms of signal selection efficiency and $b \bar{b}$ purity. As expected, the cut approach performs worse than the other two, while the best performance is observed for the pairing likelihood analysis, which was optimised for maximum purity. At the working points (see caption of Fig. 2), a gain of roughly $5 \%$ in purity is observed for the likelihood-based approaches with respect to the cut analysis at slightly higher signal selection efficiencies. All analyses suffer from a low $b \bar{b}$ purity mainly due to $b$-jet misassignments between the $t \bar{t}$ and $H$ systems. In addition, both likelihoodbased analyses enhance the signal significance with respect to the cut-based approach.

The total significance, however, depends on the systematic uncertainties of the background 


\begin{tabular}{l|ccc}
\hline & Cuts & Pairing likelihood & Constrained fit \\
\hline \hline$\sigma_{\text {tot. }}^{\text {signal }} \times B R(H \rightarrow b \bar{b})[\mathrm{fb}]$ & 517 & 517 & 517 \\
\hline$\sigma_{\text {acc. }}^{\text {signal }}[\mathrm{fb}]$ & 1.0 & 1.2 & 1.3 \\
$S / B$ & 0.11 & 0.10 & 0.12 \\
$S / \sqrt{B}$ for $30 \mathrm{fb}^{-1}$ & 1.82 & 1.95 & 2.18 \\
Irred. BG contribution & $46 \%$ & $45 \%$ & $50 \%$ \\
\hline Higgs boson jets correct & $29.4 \%$ & $34.0 \%$ & $32.0 \%$ \\
Four $b$-jets correct & $23.3 \%$ & $27.5 \%$ & $27.1 \%$ \\
Higgs boson mass resolution $[\mathrm{GeV}]$ & 22.8 & 20.1 & 22.3 \\
\hline
\end{tabular}

Table 1: Comparison of the working points of the cut based, pairing likelihood and constrained fit analyses. $\sigma_{\text {tot. }}^{\text {signal }} \times B R(H \rightarrow b \bar{b})$ denotes the total signal cross section, including the probability for the decay $H \rightarrow b \bar{b}$, $\sigma_{\text {acc. }}^{\text {signal }}$ the accepted signal cross-section after cuts.
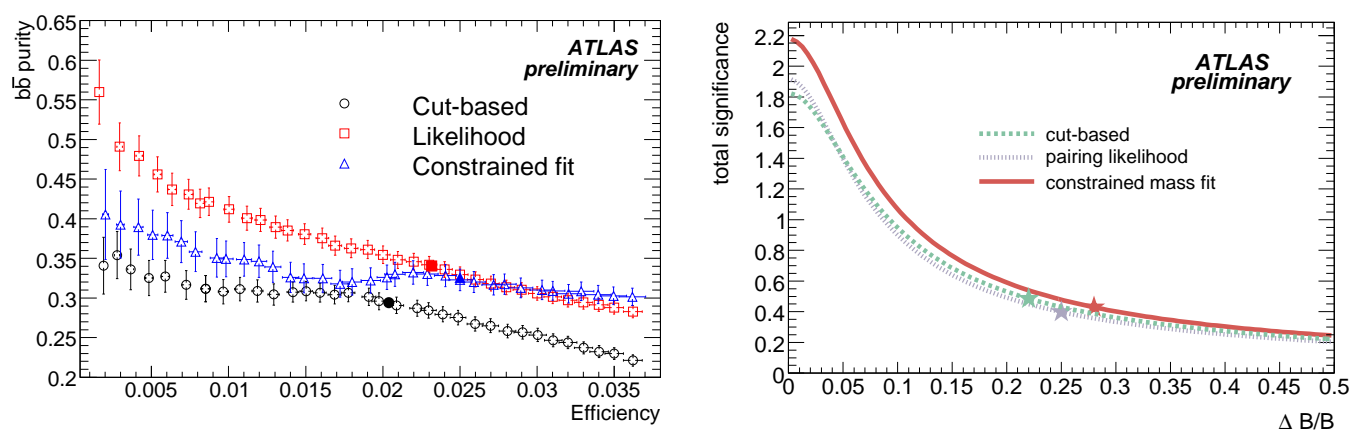

Figure 2: (Left): Comparison of the purity of the $b \bar{b}$-system attributed to the Higgs boson decay vs. the signal selection efficiency for the three analyses (see text). The solid markers show the selected working points. (Right): Total significance at an integrated luminosity of $30 \mathrm{fb}^{-1}$ as function of the relative systematic background uncertainty, $\Delta B / B$, for the three approaches. The markers indicate the significances corresponding to the estimated detector performance uncertainties.

estimation. Detector performance uncertainties of the order of $\sim 25 \%$ were estimated for the background, with the main contributions coming from jet measurement and $b$-tagging uncertainties [1]. For the current Monte Carlo based approach the largest contribution certainly is the theory uncertainty on the background. Monte Carlo driven background estimation is therefore not feasible and strategies to determine the background from data are under study. Fig. 2 (right) shows the strong dependence of the total significance on the background uncertainty, $\Delta B / B$, such that accuracies at the level of few percent are needed for the background determination in order to achieve sensitivity in the order of $2 \sigma$ and enable coupling measurements.

\section{3. $W W$ final states}

Cut based feasibility studies have been performed for the $t \bar{t} H, H \rightarrow W W$ and $W H, H \rightarrow W W$ channels. In the former case the final states containing either two like-sign isolated leptons (electrons or muons) and six jets or three leptons and four jets have been studied. The production of $t \bar{t}+$ jets was found to be the main background for both final states, where one lepton originates from a heavy quark decay. Powerful lepton isolation tools are therefore crucial for the analysis of these 
channels. Other relevant backgrounds are $t \bar{t} W, t \bar{t} Z$, $t \bar{t} t \bar{t}$ and $t \bar{t} b \bar{b}$. Due to limited statistics, jet production from QCD processes, $W t, W Z$ and $W b \bar{b}$ could not be studied in detail. The study resulted in a signal selection efficiency of $0.38 \%$ for the two lepton- and $0.17 \%$ for the three lepton analysis with respect to the initial cross-section (including the probability of the decay $H \rightarrow W W$ ) of $484 \mathrm{fb}$ for a Higgs boson mass $M_{H}=160 \mathrm{GeV}$.

The most promising final state in case of $W H$ production is the fully leptonic one. Although the corresponding branching fraction is low, the clear signature of three isolated leptons, accompanied by low jet activity and $\mathscr{E}_{T}$ results in a signal-to-background ratio of 0.75 . However, this is achieved with a low signal rate due to the low selection efficiency of $0.17 \%$ on the initial $184 \mathrm{fb}$ at a Higgs mass of $170 \mathrm{GeV}$. The main background is again $t \bar{t}$, but also $W Z$ production is sizable. Smaller contributions result from $Z Z$ and $t \bar{t} W$ production. Backgrounds from $W W W, t \bar{t} Z, W b \bar{b}$ and $W+$ jets have not yet been considered.

The results of the selections for all final states for the most promising Higgs boson masses are summarised in Tab. 2.

\begin{tabular}{l|cccccc|c}
\hline & $\sigma_{\text {acc. }}^{\text {signal }}[\mathrm{fb}]$ & $\sigma_{\text {acc. }}^{t \bar{t}}[\mathrm{fb}]$ & $\sigma_{\text {acc. }}^{t \bar{t} Z}[\mathrm{fb}]$ & $\sigma_{\text {acc. }}^{t \bar{t} W}[\mathrm{fb}]$ & $\sigma_{\text {acc. }}^{W Z}[\mathrm{fb}]$ & $\sigma_{\text {acc. }}^{\text {totalog }}[\mathrm{fb}]$ & $S / B$ \\
\hline \hline$t \bar{t} H, H \rightarrow W W(2 \mathrm{~L})$ & 1.85 & 7.4 & 1.1 & 1.7 & - & 10.3 & 0.19 \\
$t \bar{t} H, H \rightarrow W W(3 \mathrm{~L})$ & 0.82 & 2.1 & 0.9 & 0.5 & - & 3.4 & 0.24 \\
$W H, H \rightarrow W W(3 \mathrm{~L})$ & 0.31 & 0.34 & - & 0 & 0.10 & 0.45 & 0.75 \\
\hline
\end{tabular}

Table 2: Initial (including the branching ratio of the decay $H \rightarrow W W$ ) and accepted signal and background cross-sections and $S / B$ for the two lepton- (2L) and three-lepton (3L) final states of the $H \rightarrow W W$ analyses $\left(M_{H}=160 \mathrm{GeV}\right.$ for $t \bar{t} H, M_{H}=170 \mathrm{GeV}$ for $\left.W H\right)$. If no numbers are given, the background was not considered in the corresponding analysis.

\section{Summary}

For the analysis of $t \bar{t} H$, with $t \bar{t} \rightarrow \ell v q q^{\prime} b \bar{b}$ and $H \rightarrow b \bar{b}$, cut- and likelihood based approaches to separate signal and backgrounds have been investigated, leading to accepted cross-sections of about $1 \mathrm{fb}$ at signal-to-background ratios of roughly $10 \%$. The reconstruction of the Higgs mass did not produce a distinct mass peak due to limited jet resolution and wrong assignments on top of a large background. The systematic errors on a Monte Carlo driven background subtraction turned out to be large and strongly dilute the signal significance. The background therefore needs to be estimated from data with high accuracy, which is currently investigated in more detail.

In case of $W W$ final states, small signals have to be dealt with and it is not possible to reconstruct the Higgs boson mass. Also in this case background estimation from Monte Carlo will not be sufficient, and strategies need to be established to determine the background from data, which makes the analysis of these channels a challenge.

\section{References}

[1] ATLAS collaboration, Expected Performance of the ATLAS Experiment, Detector, Trigger and Physics, CERN-OPEN-2008-020, Geneva (2008), to appear.

[2] L. Vacavant, b-tagging with ATLAS, Physics at LHC, Split (2008), proceedings, this volume. 